\title{
Mitochondrial dynamics and their intracellular traffic in porcine oocytes
}

\author{
T. Yamochi ${ }^{1}$, S. Hashimoto ${ }^{2}$, A. Amo ${ }^{3}$, H. Goto ${ }^{2}$, M. Yamanaka ${ }^{2}$, M. Inoue ${ }^{2}$, Y. Nakaoka ${ }^{2}$ \\ and Y. Morimoto ${ }^{2,3}$ \\ IVF Namba Clinic, Nishik-ku; and HORAC Grand Front Osaka Clinic, Kita-ku, Osaka, Japan
}

Date submitted: 12.12.2014. Date revised: 11.05.2015. Date accepted: 03.07.2015

\section{Summary}

\begin{abstract}
Meiotic maturation of oocytes requires a variety of ATP-dependent reactions, such as germinal vesicle breakdown, spindle formation, and rearrangement of plasma membrane structure, which is required for fertilization. Mitochondria are accordingly expected be localized to subcellular sites of energy utilization. Although microtubule-dependent cellular traffic for mitochondria has been studied extensively in cultured neuronal (and some other somatic) cells, the molecular mechanism of their dynamics in mammalian oocytes at different stages of maturation remains obscure. The present work describes dynamic aspects of mitochondria in porcine oocytes at the germinal vesicle stage. After incubation of oocytes with MitoTracker Orange followed by centrifugation, mitochondria-enriched ooplasm was obtained using a glass needle and transferred into a recipient oocyte. The intracellular distribution of the fluorescent mitochondria was then observed over time using a laser scanning confocal microscopy equipped with an incubator. Kinetic analysis revealed that fluorescent mitochondria moved from central to subcortical areas of oocytes and were dispersed along plasma membranes. Such movement of mitochondria was inhibited by either cytochalasin B or cytochalasin D but not by colcemid, suggesting the involvement of microfilaments. This method of visualizing mitochondrial dynamics in live cells permits study of the pathophysiology of cytoskeleton-dependent intracellular traffic of mitochondria and associated energy metabolism during meiotic maturation of oocytes.
\end{abstract}

Keywords: Microfilament, Mitochondria, Microtubule, Oocyte, Porcine

\section{Introduction}

Meiotic maturation of oocytes involves a variety of events: germinal vesicle breakdown (GVBD), chromosomal rearrangement, the formation of the spindle and polar body, and movement of cortical granules to the subcortical areas of plasma membranes that requires structural and functional rearrangements suitable for fertilization. Given that all these events require large amounts of energy, mitochondria are expected to be localized at sites of ATP consumption. The

\footnotetext{
${ }^{1}$ All correspondence to: T. Yamochi. IVF Namba Clinic, $1-$ 17-28 Minamihorie, Nishik-ku, Osaka, Japan. Tel: +81 66534 8824. Fax: +81 66534 8876. E-mail: Yamochi@ivfnamba.com ${ }^{2}$ IVF Namba Clinic, 1-17-28 Minamihorie, Nishik-ku, Osaka, Japan.

${ }^{3}$ HORAC Grand Front Osaka Clinic, 3-1 Ofukacho, Kita-ku, Osaka, Japan.
}

intracellular localization of mitochondria in somatic cells changes markedly, depending on cell cycle and metabolic demand (Furuyama et al., 1997; Ricquier \& Bouillaud, 2000; Lee et al., 2007; Hirokawa et al., 2010). Mitochondrial dynamics and their relationships with motor proteins and the cytoskeleton in neuronal and other somatic cells have been well documented (Morris \& Hollenbeck, 1995; Kim et al., 2007; Quintero et al., 2009; Hirokawa et al., 2010; Förtsch et al., 2011). Failure of mitochondrial traffic in neurons leads to cell injury and death (Kim et al., 2007; Lee et al., 2007; Hirokawa et al., 2010). Knockout mice lacking kif5B, a member of the motor protein superfamily, displayed embryonic lethality caused by neuronal injury associated with perinuclear aggregation of lysosomes and mitochondria (Tanaka et al., 1998). Both kif1B-knockout mice and mice expressing mutant kif1B showed perinatal lethality because of respiratory failure and peripheral neuropathy (Zhao et al., 2001; 
Niwa et al., 2008). These observations suggest the importance of the role of cytoskeleton and motor proteins in localizing mitochondria appropriately at sites of energy requirement.

Mammalian oocytes have larger cytoplasm volumes and numbers of mitochondria than somatic cells (Cotterill et al., 2013). Mitochondrial distribution changes markedly during meiotic maturation of oocytes (Stojkovic et al., 2001; Sun et al., 2001; Wilding et al., 2001; Brevini et al, 2005; Liu et al., 2010; Yu et al., 2010; Ou et al., 2012). Furthermore, Brevini et al. (2005) reported that mitochondrial localization at the metaphase II (MII) stage reflected the developmental competence of oocytes. However, the dynamic aspects of mitochondria and their relationship with the cytoskeleton in oocytes appear to differ from one species to another. Mitochondria in mouse oocytes are distributed preferentially around the perinuclear area and show aggregated structures at their GV stage. However, mitochondria disperse homogeneously throughout the cytoplasm with concomitant disappearance of the aggregated structure just after the occurrence of GVBD. Mitochondria in metaphase I (MI)-stage oocytes localize around the spindle and form large clusters (Yu et al., 2010). In this context, Liu et al., (2010) reported that mitochondria were distributed around the subcortical area in GV-stage oocytes but disperse homogeneously throughout the cytoplasm at the MII stage. Microtubules, but not microfilaments, have been postulated to play a role in the mechanism of mitochondrial localization in mouse, pig, and human oocytes (Van Blerkom, 1991; Sun et al., 2001; Sun et al., 2001; Brevini et al., 2005; Calarco, 2005; Liu et al., 2010). Yu et al. (2010) reported the relationship between subcortical microfilaments and mitochondrial aggregation in mouse oocytes. Duan et al. (2014) reported that ROCK-mediated assembly of actins underlies the mechanism of mitochondrial distribution around that spindle at the MI stage of mouse oocytes. Furthermore, microfilaments have been shown to play important roles in the dynamics of cortical granules (Connors et al., 1998) and in the positioning of the meiotic spindle in oocytes (Schuh \& Ellenberg, 2008; Yi et al., 2011). These observations suggest that the cytoskeleton-dependent cellular traffic of mitochondria and other organelles in maturing oocytes differs appreciably from one species to another. To evaluate the full scope of intracellular traffic of mitochondria and its relationship with the cytoskeleton in mammalian oocytes, we developed a novel method for analyzing mitochondrial dynamics in maturing oocytes. The present report describes the dynamic behaviour of fluorescencelabelled mitochondria injected into GV-stage porcine oocytes. Kinetic analysis using inhibitors of cytoskeleton revealed that mitochondria movement in porcine oocytes depends strongly on the microfilament network.

\section{Materials and methods}

\section{Oocytes collection}

Porcine ovaries were freshly obtained from prepubertal gilts at a local slaughterhouse and cumulusoocyte complexes (COCs) were obtained by aspiration of their follicles (3-6 $\mathrm{mm}$ in diameter) using a 21-gauge needle and a $10-\mathrm{ml}$ syringe. COCs that had multiple layers of compacted cumulus cells were selected. COCs were suspended in 199 Hank's salt solution (Life Technologies, Carlsbad, CA, USA) containing $0.1 \%(\mathrm{w} / \mathrm{v}$ ) hyaluronidase (Sigma Chemical Co., St Louis, MO, USA) for 10 min using a vortex to remove cumulus cells. Denuded oocytes were washed three times with culture medium for porcine oocytes (POM; Yoshioka et al., 2008) containing $0.02 \mathrm{AU} \mathrm{ml}^{-1}$ follicle stimulating hormone (Kyoritsuseiyak, Tokyo, Japan), $10 \mathrm{IU} \mathrm{ml} \mathrm{m}^{-1}$ human chorionic gonadotropin (Aska Pharmaceutical Co., Ltd, Tokyo, Japan), $25 \mathrm{mmol} / 1$ 2-mercaptoethanol (Sigma), $1 \mu \mathrm{g} \mathrm{ml} \mathrm{m}^{-1} \beta$-estradiol (Sigma), and $0.3 \%(\mathrm{w} / \mathrm{v})$ bovine serum albumin (Sigma) and then cultured in the same medium at $38.5^{\circ} \mathrm{C}$ under $5 \% \mathrm{CO}_{2}, 5 \% \mathrm{O}_{2}$, and $90 \% \mathrm{~N}_{2}$.

\section{Mitochondria and endoplasmic reticulum}

Mitochondria and endoplasmic reticulum (ER) in oocytes were stained with $10 \mu \mathrm{M}$ MitoTracker Orange CM-H2TMRos (MTO; Life Technologies) or $1 \mu \mathrm{M}$ ER Tracker Red (ERT; Life Technologies) at $37^{\circ} \mathrm{C}$ for $30 \mathrm{~min}$. After washing three times with Hank's 199 medium, mitochondria- or ER-stained oocytes were observed under a laser scanning confocal microscope capable of time-lapse cinematography (CV1000, Yokogawa Electric, Tokyo, Japan). Some stained oocytes were used as donor cells for mitochondrial transfer or ER transfer. Mitochondria and ER were concentrated in cytoplasm of oocytes by centrifugation at $10,000 \mathrm{~g}$ and $37^{\circ} \mathrm{C}$ for $15 \mathrm{~min}$ as described by Ferreira et al. (2010). After centrifugation, oocytes were transferred into a drop of $10 \mu l 199$ Hank's containing $5 \mu \mathrm{g}^{-1}$ cytochalasin B (Sigma); fluorescently labelled mitochondria were effectively concentrated in a heavy fraction of oocytes (Fig. 1). Mitochondria- or ER-enriched ooplasm was aspirated into a micropipette and transferred into recipient oocytes in either their central or subcortical areas using a pair of micromanipulators (Narishige, Tokyo, Japan) on an inverted microscope (IX71; Olympus, Tokyo, Japan). 


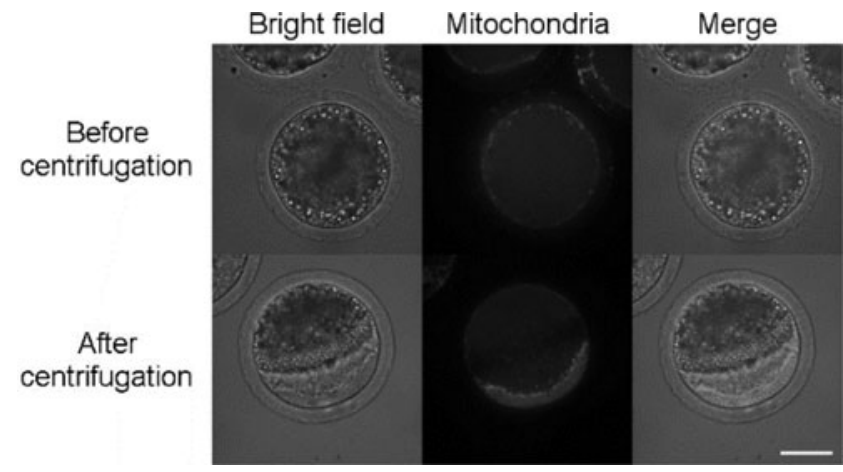

Figure 1 Mitochondrial localization in porcine oocytes before and after centrifugation. Porcine oocytes were incubated in 199 Hank's salt containing $10 \mu \mathrm{M}$ MitoTracker Orange (MTO) at $37^{\circ} \mathrm{C}$ for $30 \mathrm{~min}$. After staining mitochondria with MTO, oocytes were centrifuged at $10,000 \mathrm{~g}$ at $37^{\circ} \mathrm{C}$ for $15 \mathrm{~min}$. Before and after centrifugation, oocytes were observed under a confocal fluorescence microscope. Bar $=50 \mu \mathrm{m}$.

\section{Analysis by time-lapse cinematography}

MitoTracker- or ER-stained oocytes and recipient oocytes injected with fluorescence-labelled mitochondria or ER were observed under a laser scanning confocal microscope capable of time-lapse cinematography. The oocytes were transferred to a drop of $5 \mu \mathrm{l}$ POM on a glass dish (MatTek Corporation, Ashland, MA, USA) and placed in an incubation chamber at $37^{\circ} \mathrm{C}$ under $90 \% \mathrm{~N}_{2}, 5 \% \mathrm{CO}_{2}$, and $5 \% \mathrm{O}_{2}$. Fluorescence images of oocytes were obtained every $15 \mathrm{~min}$ for $15 \mathrm{~h}$. To determine the possible involvement of the cytoskeleton in mitochondrial traffic, their dynamics in the presence of either, $0.1 \mu \mathrm{g} \mathrm{ml}^{-1}$ colcemid (Life Technologies), $5 \mu \mathrm{g} \mathrm{ml} \mathrm{m}^{-1}$ cytochalasin $\mathrm{B}$, or $2.5 \mu \mathrm{g}$ $\mathrm{ml}^{-1}$ cytochalasin D (Sigma) were also analyzed. Mitochondrial dynamics in the recipient oocytes were analyzed qualitatively and quantitatively with ImageJ software. Relative area, length, and distribution of fluorescent mitochondria in recipient oocytes were calculated on the basis of their initial values at $0 \mathrm{~h}$ of culture. To quantitate mitochondrial movement, timedependent changes in the cytoplasmic areas occupied by fluorescence-labelled mitochondria injected either centrally or subcortically in oocytes were measured every $1 \mathrm{~h}$ (see Fig. 2).

\section{Immunological staining of the cytoskeleton}

After time-lapse analysis, microtubules and microfilaments in oocytes were visualized by specific staining. Oocytes were fixed for microtubule analysis as described by Albertini \& Clark (1984) with minor modifications. To stain microtubules, oocytes were fixed in microtubule stabilization buffer (MTSB-XF) containing 0.1 M PIPES (Sigma), $5 \mathrm{mM} \mathrm{MgCl}_{2}$ (Wako, Osaka, Japan), $2.5 \mathrm{mM}$ EGTA (Sigma), 0.01\% (v/v)

\section{A Before culture}
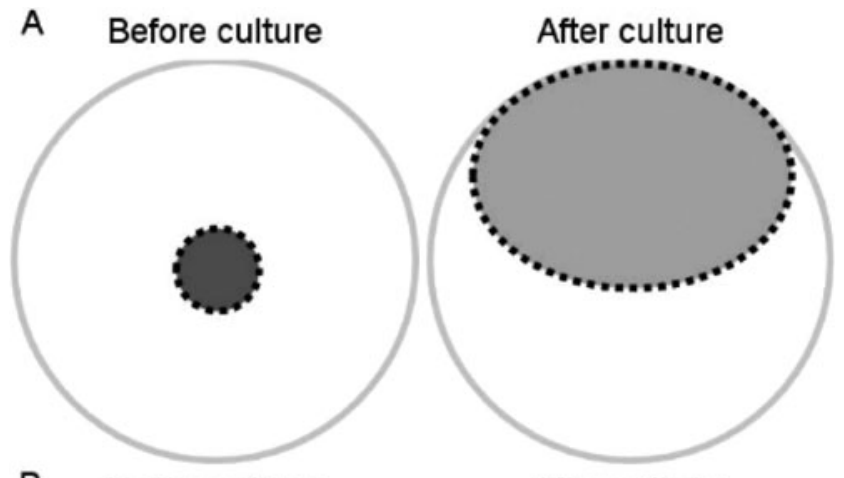

B Before culture
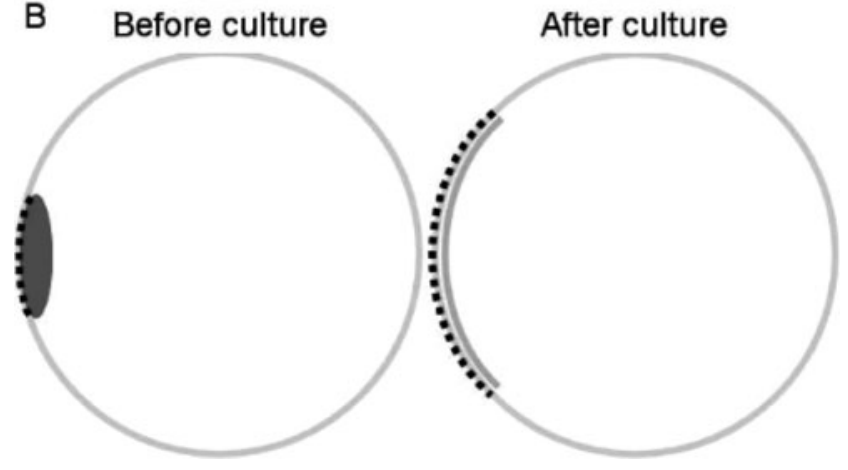

Figure 2 Scheme of mitochondrial dynamics. Mitochondria were injected at central $(A)$ or subcortical areas $(B)$ in oocytes. The dark grey field and grey solid line show the injected mitochondria and plasma membranes, respectively. To analyze the dynamics of the injected mitochondria, timedependent changes in the area enclosed by the dotted black line $(A)$, and in the length shown by the dotted black line $(B)$ were measured every $1 \mathrm{~h}$ with Image $\mathrm{J}$ software.

aprotinin (Sigma), $1 \mathrm{mM}$ dithiothreitol (Sigma), 50\% (v/v) deuterium oxide (Sigma), $1 \mu \mathrm{M}$ paclitaxel (Sigma), 0.1\% (v/v) Triton X-100 (Sigma), and 2\% (v/v) formaldehyde (Sigma) at $4^{\circ} \mathrm{C}$ overnight. The fixed oocytes were blocked with phosphate-buffered saline (PBS) $(-)$ containing $1 \%(\mathrm{w} / \mathrm{v})$ bovine serum albumin (BSA) at $37^{\circ} \mathrm{C}$ for $1 \mathrm{~h}$ and then treated with Alexa 488-conjugated anti- $\alpha$-tubulin mouse antibody (Life Technologies) at $4^{\circ} \mathrm{C}$ for $1 \mathrm{~h}$ followed by washing and counterstaining with $10 \mu \mathrm{g} \mathrm{ml}^{-1}$ Hoechst33342 (Dojindo, Kumamoto, Japan) at $25^{\circ} \mathrm{C}$ for $10 \mathrm{~min}$. Immunostained oocytes were placed on a slide glass and mounted with a small drop of Vectashield mounting medium (Vector Laboratories, Burlingame, CA, USA) for analysis with a laser scanning confocal microscope. To stain microfilaments, oocytes were also fixed with $2 \%(\mathrm{v} / \mathrm{v})$ paraformaldehyde (Wako) in PBS (-) at $4^{\circ} \mathrm{C}$ overnight. The fixed oocytes were permeabilized with $0.1 \%$ Triton $\mathrm{X}-100(\mathrm{v} / \mathrm{v})$ in PBS at $37^{\circ} \mathrm{C}$ for $45 \mathrm{~min}$ followed by the permeabilization step and then incubated in blocking solution containing $0.1 \mu \mathrm{g} \mathrm{ml}^{-1}$ FITC-conjugated phalloidin (Sigma) at $37^{\circ} \mathrm{C}$ for $1 \mathrm{~h}$. After repeated washing, oocytes were 

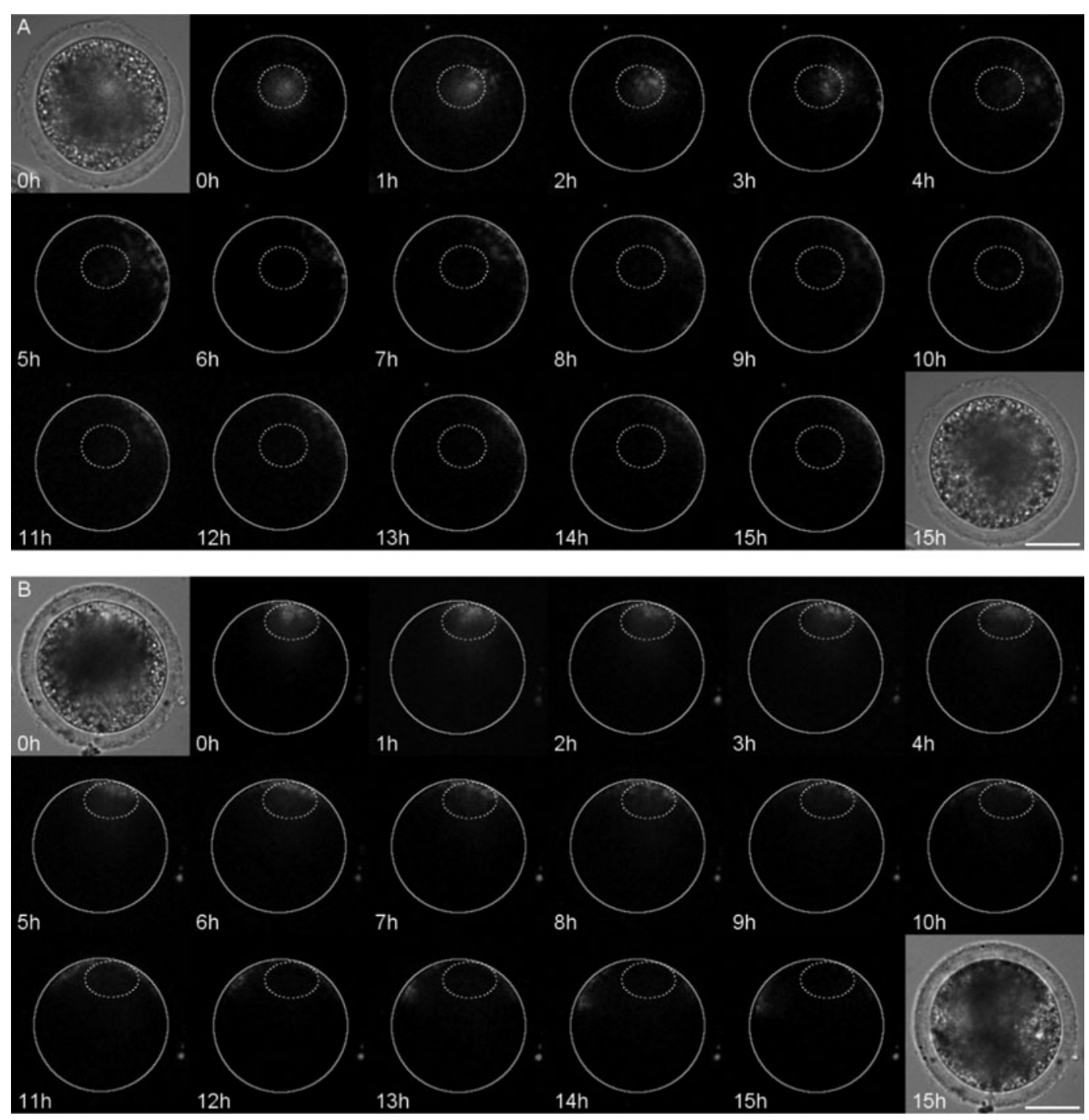

Figure 3 Dynamic aspects of fluorescence-labelled mitochondria injected in porcine oocytes. Fluorescence-labelled mitochondria were obtained from MitoTracker Orange (MTO)-stained oocytes and injected in recipient oocytes. Time-lapse cinematographic images were obtained from oocytes after injection of mitochondria in either the central $(A)$ or subcortical area $(B)$. Injected mitochondria moved from the central portion to the subcortical area. After accession of subcortical area, injected mitochondria showed isotropic movement along plasma membrane $(A)$. Peripherally injected mitochondria moved topographically along plasma membranes $(B)$. Note that mitochondrial fluorescence was detected preferentially along the subcortical area. Solid and dotted lines indicate plasma membranes and mitochondria-injected areas in recipient oocytes, respectively. Bar $=50 \mu \mathrm{m}$.

counterstained with $10 \mu \mathrm{g} \mathrm{ml}{ }^{-1}$ Hoechst 33342 in blocking solution at $25^{\circ} \mathrm{C}$ for $10 \mathrm{~min}$. Oocytes thus treated were transferred to a $5 \mu \mathrm{l}$ drop of PBS (-) on a glass dish and observed with a laser scanning confocal microscope.

\section{Statistical analysis}

The dynamics of fluorescence-labelled mitochondria in oocytes were analyzed by Tukey-Kramer test. A $P$-value less than 0.05 was considered statistically significant.

\section{Results}

\section{Mitochondrial distribution and dynamics in porcine oocytes}

To investigate the intracellular traffic of mitochondria, porcine oocytes were incubated with MitoTracker, and the dynamic aspect of fluorescence-labelled mitochondria was observed. Although fluorescencelabelled mitochondria distributed widely throughout the cytoplasm of oocytes, confocal microscopic analysis revealed that the fluorescence intensity 


\section{Bright field Mitochondria}

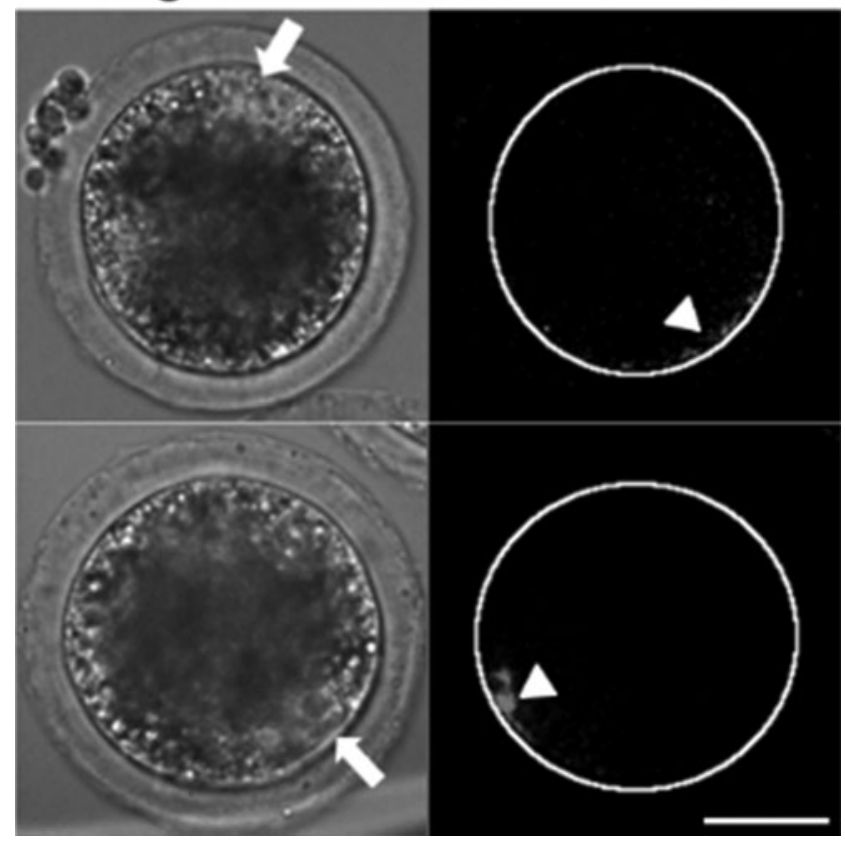

Figure 4 Position of germinal vesicle (GV) nucleus of recipient oocytes at the time of injected mitochondria arriving subcortical area. Representative images of mitochondriainjected oocytes at the time of injected mitochondria arriving subcortical area. Recipient oocytes were injected with mitochondria-enriched ooplasm, and were cultured in same conditions of Fig. 3. Note that the injected mitochondria moved toward subcortical area of plasma membrane independently from the position of GV nucleus. Arrows and arrowheads indicate the position of GV nucleus and injected mitochondria, respectively. Bar $=50 \mu \mathrm{m}$.

was stronger in the subcortical area than in the central area (Fig. S1). To characterize mitochondrial dynamics in oocytes, the fluorescence-labelled mitochondria were injected into recipient cells. Time-lapse cinematographic analysis revealed that fluorescencelabelled mitochondria injected in central areas of oocytes moved towards the subcortical area close to plasma membranes. The injected mitochondria moved isotropically along plasma membranes after arriving to subcortical areas. When injected in the subcortical area, mitochondria preferentially moved laterally along plasma membranes (Fig. 3). We also analyzed the relationship between the direction of movement of the centrally injected mitochondria and the position of GV nucleus. Our result showed that mitochondria moved towards the subcortical area of plasma membrane independently from the position of GV nucleus (Fig. 4). Such movement and redistribution of the injected mitochondria were not observed with degenerated oocytes, irrespective of the site of injection (data not shown).
Kinetic analysis in the presence of inhibitors of the cytoskeleton

To examine the relationship between mitochondrial traffic and the cytoskeleton in porcine oocytes, we tested the effects of inhibitors of the cytoskeleton on the movement of mitochondria. The presence of colcemid, an inhibitor of microtubules, did not appreciably inhibit the movement and redistribution of fluorescence-labelled mitochondria injected in either the central or subcortical area (Fig. 5). In contrast, the presence of either cytochalasin B or cytochalasin D, inhibitors of microfilaments, strongly inhibited the movement and redistribution of mitochondria, irrespective of the site of injection in oocytes (Fig. 6 and Fig. 7).

\section{Immunocytochemistry of the cytoskeleton in oocytes}

To confirm the effects of inhibitors on the cytoskeleton, microtubules and microfilaments in mitochondriainjected oocytes were immunostained after treatment of cells with either colcemid, cytochalasin B, or cytochalasin D. Control oocytes showed typical networks of microtubules in their cytoplasm. Networks were strongly disrupted by treatment of oocytes with colcemid but not cytochalasin B and D. Oocytes showed typical networks of microfilaments predominantly in subcortical areas along plasma membranes. The networks were disrupted markedly by either cytochalasin B or D, but not by colcemid (Fig. 8).

\section{Quantitation of mitochondrial dynamics}

To quantify the dynamics of the injected mitochondria, we measured time-dependent changes in the fluorescence-positive area or the length as described in Fig. 2. During $15 \mathrm{~h}$ of culture, the area and the length occupied by fluorescence-labelled mitochondria increased time dependently irrespective of the sites of injection, by some mechanism that was strongly inhibited by either cytochalasin B or D, but not colcemid (Fig. 9).

\section{Dynamics of fluorescence-labelled ER injected in oocytes}

To examine whether the observed movement was mitochondria specific or not, we also injected ooplasm enriched with fluorescence-labelled ER into recipient oocytes. Fluorescent ER dispersed across the broad of the cytoplasm of recipient oocytes at kickoff point of time-lapse imaging (15-30 min after injection). However, the fluorescence intensity of ER injected into recipient oocytes rapidly decreased with time and reached to below detectable levels $1 \mathrm{hr}$ after the injection (Fig. 10). 

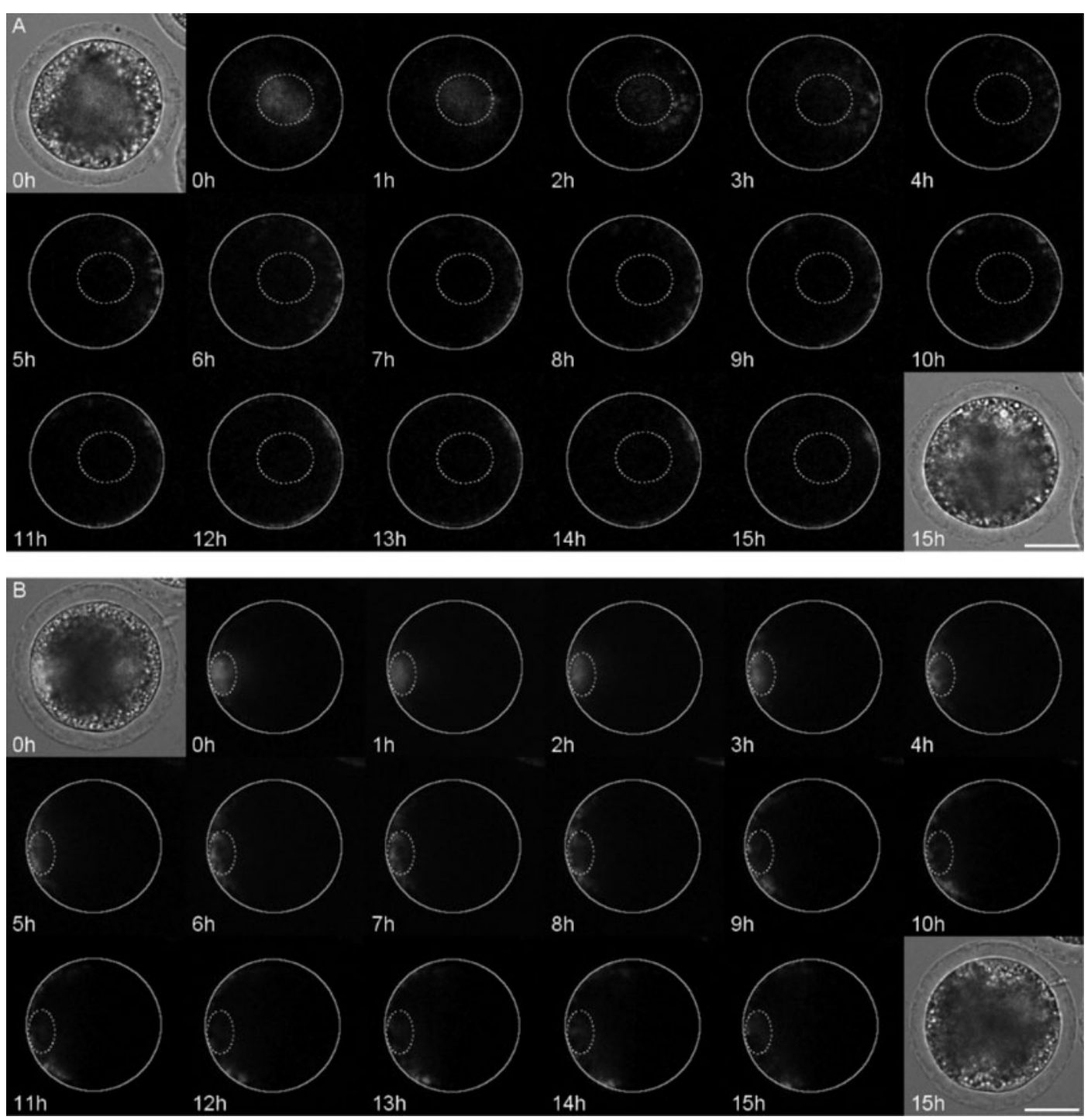

Figure 5 Effect of colcemid on mitochondrial dynamics in porcine oocytes. Time-lapse cinematographic images of the movement of injected mitochondria in colcemid- treated oocytes. Recipient oocytes were injected with mitochondria-enriched ooplasm in either the central $(A)$ or the subcortical area $(B)$ of the cells. Mitochondria-injected oocytes were treated with 0.1 $\mu \mathrm{g} / \mathrm{ml}$ colcemid at $37^{\circ} \mathrm{C}$ during time-lapse cinematographic analysis. Bar $=50 \mu \mathrm{m}$.

\section{Discussion}

Mitochondrial dynamics in mammalian oocytes differ significantly from one species to another and depends on their meiotic stages, such as GV, MI, and MII. Because mammalian oocytes have large numbers of mitochondria, it is difficult in practice to obtain precise information on their dynamics. The present work describes a method of analyzing the time-dependent movement and distribution of mitochondria in the GV stage of porcine oocytes. The results clearly showed that porcine GV oocytes have at least two types of mitochondrial traffic; one mobilizing them from the central (perinuclear) to subcortical areas and the other dispersing them along the subcortical space of plasma membranes. To avoid possible injury to mitochondria during their isolation (Barritt et al., 2001), we used mitochondria-enriched ooplasm obtained from MitoTracker-labelled oocytes as donor specimens, as described in the text. We also analyzed the dynamics of endogenously labelled mitochondria by directly injecting small volumes of high concentrations of MitoTracker into oocytes. However, the fluorescence intensity of MitoTracker accumulated in mitochondria rapidly decreased. Thus, long-term observation of their dynamics was difficult in practice. It should be noted that a mitochondrial membrane potential is required to retain the accumulated fluorescent dye in their matrix. Because MitoTracker was dissolved in high concentrations of DMSO, the amphipathic 

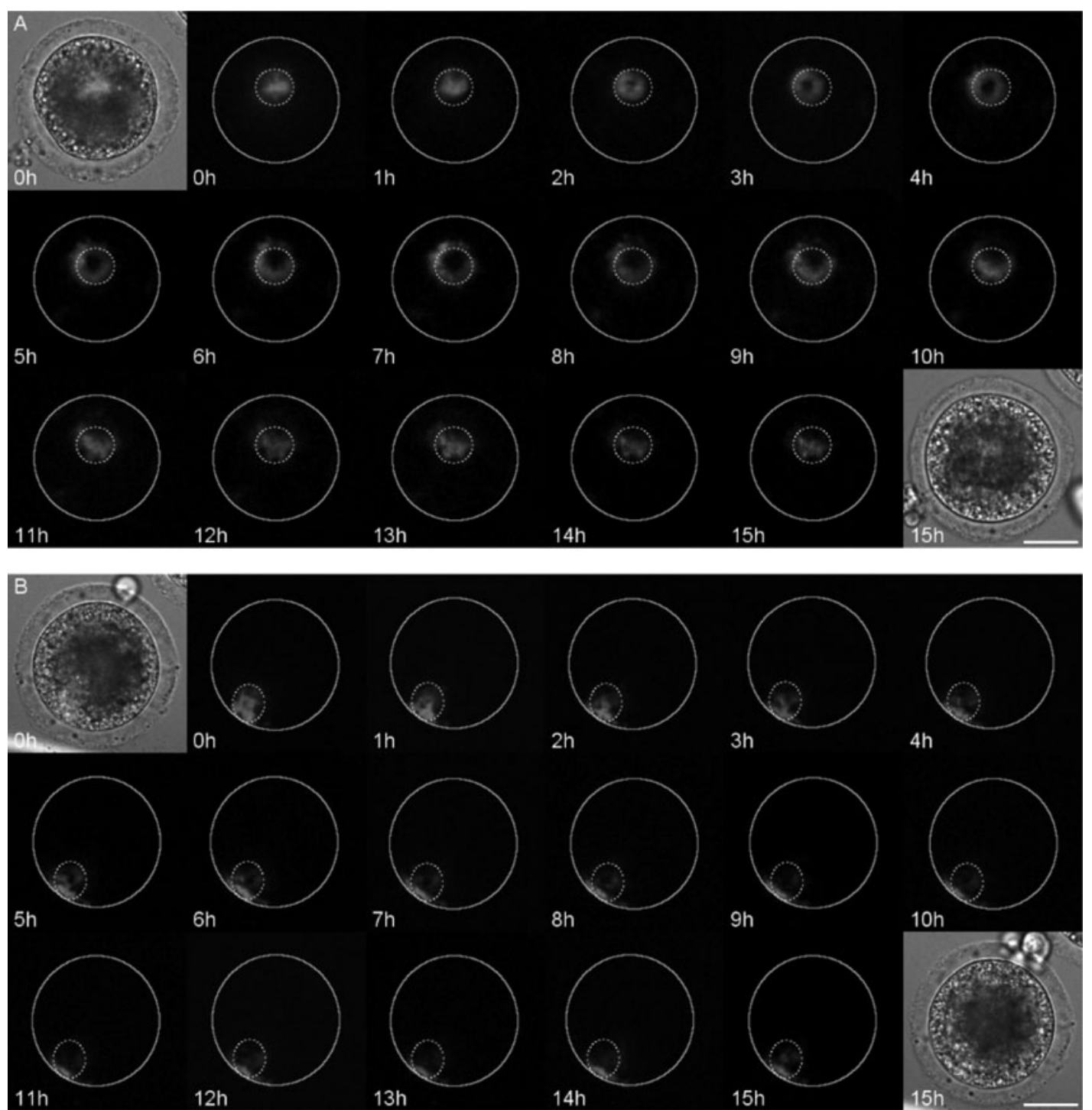

$6 \mathrm{~h}$

$7 \mathrm{~h}$

$8 \mathrm{~h}$

9h

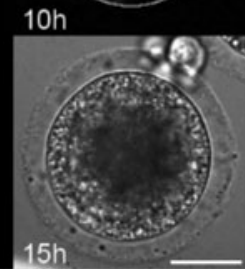

Figure 6 Effect of cytochalasin B on mitochondrial dynamics in porcine oocytes. Time-lapse cinematographic images of mitochondrial movement were obtained with cytochalasin B-treated oocytes. Recipient oocytes were injected with mitochondria-enriched ooplasm in either the central $(A)$ or the subcortical area $(B)$ of the cells. Mitochondria-injected oocytes were treated with $5 \mu \mathrm{g} / \mathrm{ml}$ cytochalasin $B$ at $37^{\circ} \mathrm{C}$ during the analysis. Note that the injected mitochondria remained localized at the sites of injection in cytochalasin B-treated oocytes. Bar $=50 \mu \mathrm{m}$.

nature of the solvent and/or the fluorescent dye were expected to exert toxic effects on mitochondria. This could be the reason why the fluorescent dye that directly and locally accumulated in mitochondria rapidly disappeared in oocytes. An explanation of the mechanism by which the fluorescence intensity of endogenously labelled mitochondria decreased rapidly awaits further study.

Experiments using specific inhibitors for the cytoskeleton suggested that cytochalasin-sensitive microfilaments, but not colcemid-sensitive microtubules, play critical roles in the mitochondrial traffic in porcine GV oocytes. In this context, microtubules have been postulated to play important roles in mitochondrial traffic in neurons and other somatic cells (Furuyama et al., 1997; Ricquier \& Bouillaud, 2000; Lee et al., 2007; Hirokawa et al., 2010), and during meiotic maturation of mouse, pig and human oocytes (Van Blerkom, 1991; Sun et al., 2001; Calarco, 2005; Liu et al., 2010). Van Blerkom (1991) reported that mitochondria localized evenly in the cytoplasm of early stage of mouse GV oocytes and then translocated to the chromosomal area during meiotic maturation of cells. Mitochondria that localized around subcortical areas moved to a central area of porcine oocyte during GVBD (Sun et al., 2001). In contrast, mitochondria that localized homogeneously in extracortical areas of human oocytes dispersed within cells during 

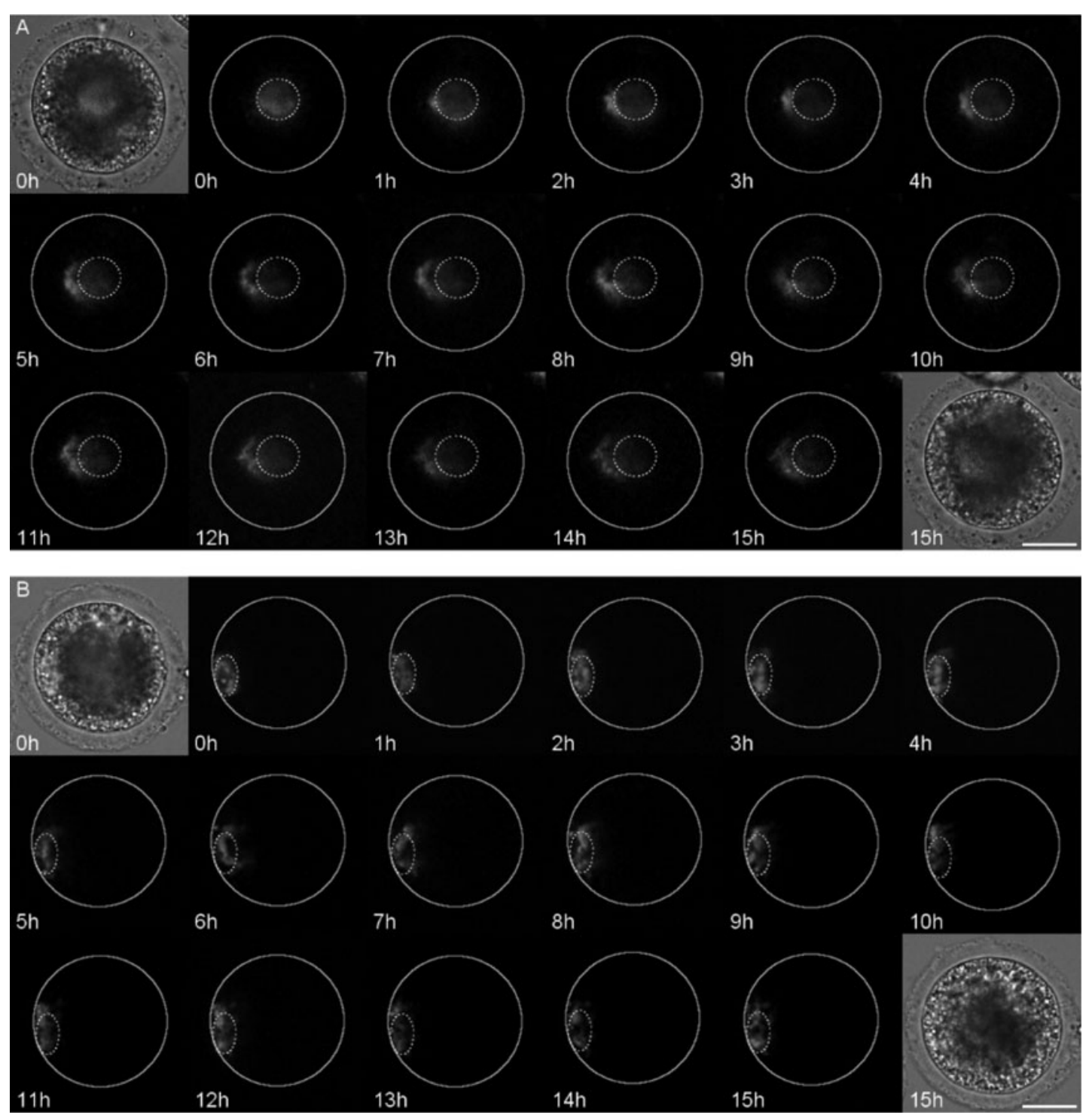

Figure 7 Effect of cytochalasin D on mitochondrial dynamics in porcine oocytes. Time-lapse cinematographic images of mitochondrial movement were obtained with cytochalasin D-treated oocytes. Recipient oocytes were injected with mitochondria-enriched ooplasm in either the central $(A)$ or the subcortical area $(B)$ of cells. Mitochondria-injected oocytes were treated with $2.5 \mu \mathrm{g} / \mathrm{ml}$ cytochalasin $\mathrm{D}$ at $37^{\circ} \mathrm{C}$ during the analysis. Note that the injected mitochondria remained localized at the sites of injection in cytochalasin D-treated oocytes. Bar $=50 \mu \mathrm{m}$.

meiotic maturation (Liu et al., 2010). Furthermore, such translocations of mitochondria in porcine and human oocytes were suppressed by specific inhibitors of microtubules (Sun et al., 2001; Liu et al., 2010). These results suggested that microtubules underlie the mechanisms for movement of mitochondria in the oocytes of these species. In contrast, microfilaments have been shown to play a role in mitochondrial movement and localization in budding yeasts, neurons and HeLa cells (Tanaka et al., 1998; Zhao et al., 2001; Boldogh \& Pon, 2006; Lee et al., 2007; Quintero et al., 2009; Förtsch et al., 2011). Furthermore, recent studies have also suggested the involvement of microfilaments in mitochondrial localization in mammalian oocytes (Yu et al., 2010; Duan et al., 2014). Yu et al. (2010) reported that the cortical network of microfilaments plays an important role in the aggregation of mitochondria in mouse oocytes. Duan et al. (2014) reported that the assembly of actins mediated by Rho-associated coiled-coil forming kinase plays critical roles in mitochondrial localization around the spindle in mouse MI oocytes. Microfilaments localize widely in cytoplasm of mammalian oocytes but densely in the subcortical area of plasma membranes (Schuh \& Ellenberg, 2008; Yi et al., 2011; Schuh, 2011). Thus, microfilaments might also play critical roles in the 


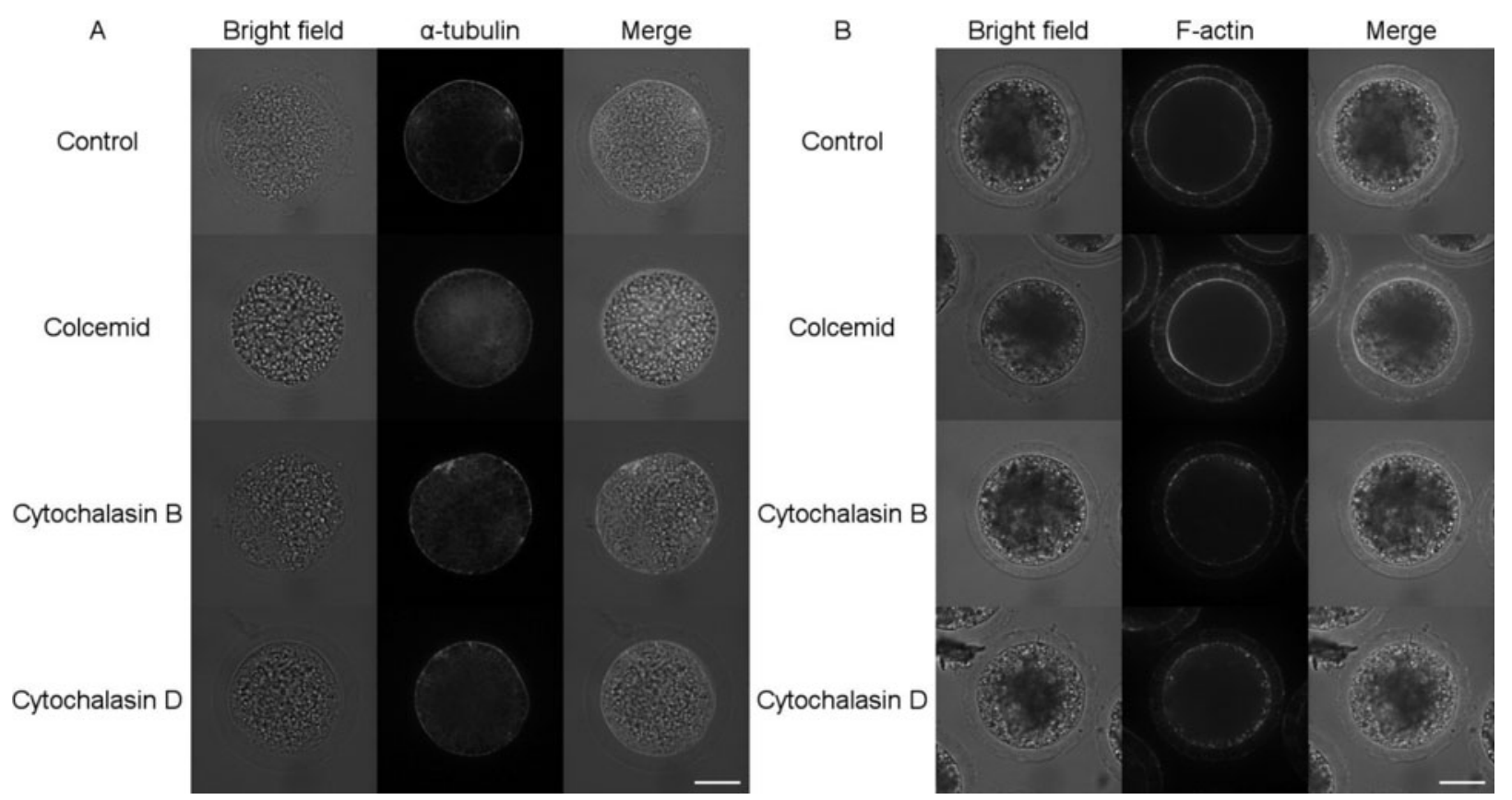

Figure 8 Effect of various inhibitors on the cytoskeletal network in porcine oocytes. Recipient oocytes were incubated in POM medium in the presence or absence of colcemid $(0.1 \mu \mathrm{g} / \mathrm{ml})$, cytochalasin B $(5 \mu \mathrm{g} / \mathrm{ml})$, or cytochalasin $\mathrm{D}(2.5 \mu \mathrm{g} / \mathrm{ml})$ at $37^{\circ} \mathrm{C}$ during the analysis. After time-lapse analysis, oocytes were fixed and stained with $\alpha$-tubulin $(A)$ or F-actin $(B)$ as described in the text. Note that colcemid selectively disrupted the microtubule network $(A)$ without affecting the microfilament network $(B)$, whereas both cytochalasin B and D specifically perturbed the subcortical network of microfilaments $(B)$ without disturbing the microtubule network $(A)$. Bar $=50 \mu \mathrm{m}$.

cytochalasin-sensitive movement of mitochondria in porcine oocytes at the GV stage observed in the present work. In this context, Sun et al. (2001) reported that microfilaments were not involved in mitochondrial movement after GVBD of porcine oocytes. Calarco (2005) also failed to show the involvement of microfilaments in mitochondrial dynamics in mouse oocytes. These observations suggest that cytoskeletons involved in the movement of mitochondria might change dynamically at the stage of meiotic maturation of oocytes.

The present work shows that centrally injected mitochondria moved unidirectionally toward subcortical areas independent of the localization of nucleus and suggests the presence of vectorial transport of mitochondria within porcine oocytes. The present work also shows isotropic movement of mitochondria along plasma membranes after arriving at subcortical areas. However, the movement of mitochondria from subcortical to central areas was not apparent under our experimental conditions. Preliminary experiments using a transmission electron microscope revealed that mitochondria distributed homogeneously in the cytoplasm of porcine oocyte at GV stage (data not shown). Thus, mitochondria seem to have both anterograde (towards plasma membranes) and retrograde (centripetal) trafficking connecting central and subcortical areas in oocytes thereby allowing homogeneous distribution in cells. In this context, neurons have both anterograde and retrograde trafficking of mitochondria; the velocity of the two movement differed significantly (Hirokawa et al., 2010). Thus, oocytes would also have both anterograde and retrograde traffics for mitochondria to support energy metabolism at different times and subcellular sites during meiotic maturation. It should be noted that anterograde and retrograde trafficking are driven by kinesin and dynein, respectively, on the railroads on cytoskeletons (Tanaka et al., 1998; Zhao et al., 2001). The present work shows that mitochondrial movement in oocytes was inhibited by cytochalasins $B$ and $D$, specific inhibitors of microfilaments. Microfilaments play important roles as the scaffold for myosin family motor proteins (Seabra \& Coudrier, 2004). Mitochondrial traffic in neurons is driven not only by kinesin and dynein, but also by myosin (Tanaka et al., 1998; Zhao et al., 2001; Quintero et al., 2009; Förtsch et al., 2011). Thus, myosins seem to underlie the mechanism of mitochondrial movement in porcine oocytes. Microfilaments have been shown to participate in cytoplasmic movement of mitochondria by interacting with actin-related protein $2 / 3$ complex in oocytes (Yi et al., 2011; Li \& Albertini, 2013). Yi et al. (2011) reported that depolymerization of actin 

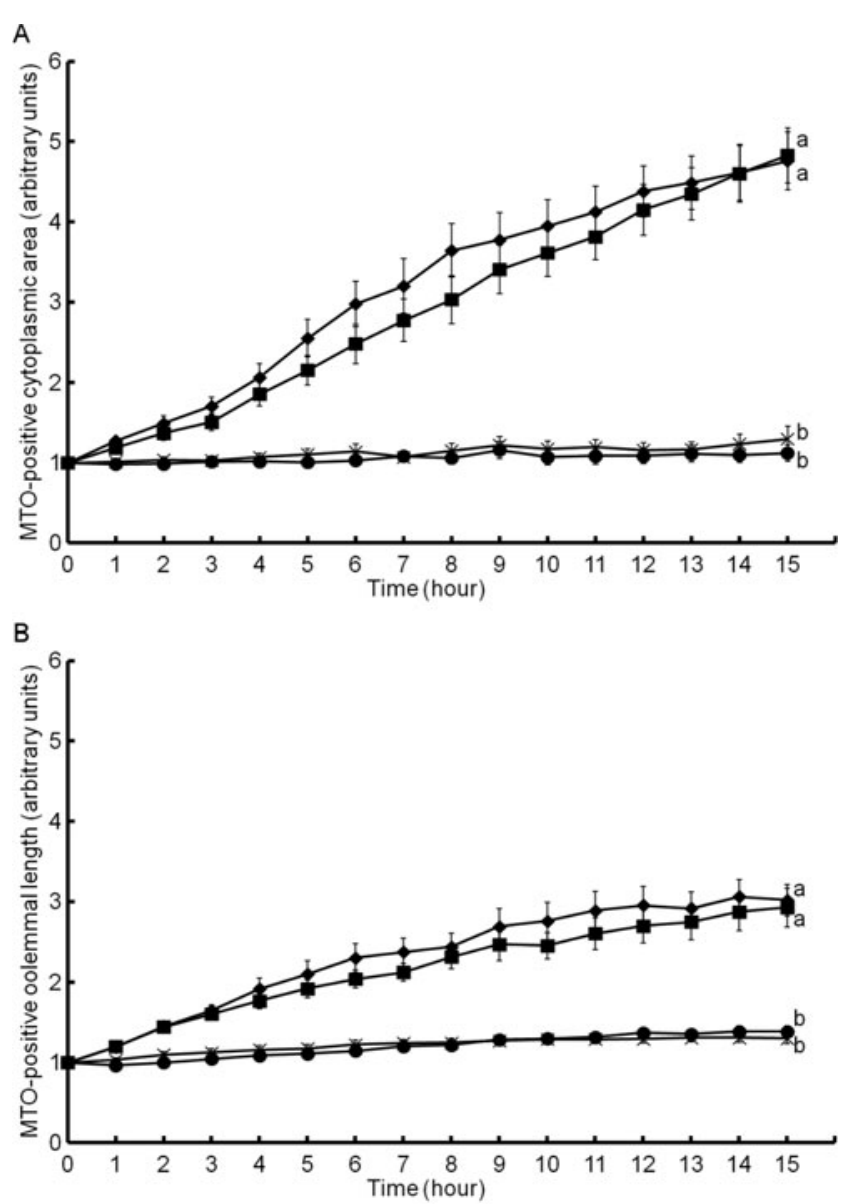

Figure 9 Time-dependent changes in the distribution of injected mitochondria. Under conditions identical to those in Figs 4, 6, 7 and 8 changes in the cytoplasmic area having fluorescence-labelled mitochondria were analyzed with oocytes in the absence $(\boldsymbol{\square})$ or presence of colcemid $(\bullet)$, cytochalasin B $(\bullet)$, or cytochalasin D $(\times)$. Values show the size of the fluorescence-labelled area relative to that of the initial values. Fluorescence-labelled mitochondria were injected in the central $(A)$ or the subcortical area $(B)$ in oocytes (central: control, $n=34$, colcemid, $n=29$, cytochalasin $\mathrm{B}, \mathrm{n}=30$, cytochalasin $\mathrm{D}, n=30$, subcortical: control, $n=26$, colcemid, $n=25$, cytochalasin $\mathrm{B}, n=28$, cytochalasin $\mathrm{D}, n=25$, respectively).

filaments abolished the cytoplasmic mobilization of mitochondria in MI oocytes. To clarify the mechanism for the 3D trafficking of mitochondria in large and non-polarized mammalian oocytes, their relationship with the cytoskeleton and motor proteins during their meiotic maturation should be studied further.

To examine whether the vectorial movement of the transferred mitochondria in oocytes was specific or not, we also injected ERT-labelled ER into oocytes and observed their movement in the cells. However, the rate of disappearance of the ER-associated fluorescence dye was fairly rapid. The stability of ERT in ER was significantly lower than that of MTO in

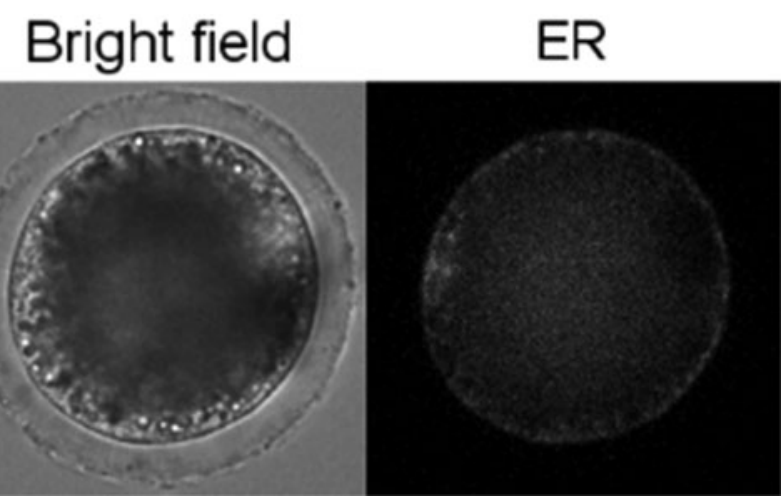

Figure 10 Distribution in fluorescent endoplasmic reticulum (ER) in recipient oocytes. The representative image of fluorescent ER-injected oocytes. Fluorescence-labelled ER were obtained from ERT-stained oocytes and injected into recipient oocytes. Images were obtained from fluorescent ERinjected oocytes at 15-30 min after injection. Bar $=50 \mu \mathrm{m}$.

mitochondria and, hence, it was difficult in practice to analyze the time-dependent movement of the injected ER in the recipient oocytes. Thus, the specificity of the observed movement of the fluorescence-labelled mitochondria that is specific for this organelle requires further study. The reason why the fluorescence intensity of ERT decreased quickly within the recipient oocytes remains unknown. Studies using other dyes that specifically accumulated in ER with prolonged duration than ERT, such as 1,1'-dioctadecyl-3,3,3',3'tetramethylindocarbocyanine perchlorate, are under our current investigation.

The method established in the present study permits examination of the pathophysiology of intracellular trafficking of mitochondria and other organelles in oocytes from patients with infertility. As mitochondria and oxidative stress have been postulated to underlie the pathogenesis of aging in a wide variety of cells including oocytes, the present method also permits studies on the mechanism by which chromosomal aberrations and other maturation failures are increased during meiotic maturation and aging of mammalian oocytes.

\section{Acknowledgements}

The authors would like to thank Enago (www.enago.jp) for the English language review.

\section{Financial support}

This research received no specific grant from any funding agency, commercial or not-for- profit sectors. 


\section{Conflict of interest}

None.

\section{Supplementary materials}

Supplementary materials are available with this paper. Please visit http://dx.doi.org/10.1017/ S0967199415000489

\section{References}

Albertini, D.F., Herman, B. \& Sherline, P. (1984). In vivo and in vitro studies on the role of HMW-MAPs in taxol-induced microtubule bundling. Eur. J. Cell. Biol. 33, 134-43.

Barritt, J., Willadsen, S., Brenner, C. \& Cohen, J. (2001). Cytoplasmic transfer in assisted reproduction. Hum. Reprod. Update 7, 428-35.

Boldogh, I.R. \& Pon, L.A. (2006). Interactions of mitochondria with the actin cytoskeleton. Biochim. Biophys. Acta 1763. 450-62.

Brevini, T.A., Vassena, R., Francisci, C. \& Gandolfi, F. (2005). Role of adenosine triphosphate, active mitochondria \& microtubules in the acquisition of developmental competence of parthenogenetically activated pig oocytes. Biol. Reprod. 72, 1218-23.

Calarco, P.G. (2005). The role of microfilaments in early meiotic maturation of mouse oocytes. Microsc. Microanal. 11, 146-53.

Connors, S.A., Kanatsu-Shinohara, M., Schultz, R.M. \& Kopf, G.S. (1998). Involvement of the cytoskeleton in the movement of cortical granules during oocyte maturation, and cortical granule anchoring in mouse eggs. Dev. Biol. 200, 103-15.

Cotterill, M., Harris, S.E., Collado, Fernandez, E., Lu, J., Huntriss, J.D., Campbell, B.K. \& Picton, H.M. (2013). The activity and copy number of mitochondrial DNA in ovine oocytes throughout oogenesis in vivo and during oocyte maturation in vitro. Mol. Hum. Reprod. 19, 444-50.

Duan, X., Liu, J., Dai, X.X., Liu, H.L., Cui, X.S., Kim, N.H., Wang, Z.B., Wang, Q. \& Sun, S.C. (2014). Rho-GTPase effector ROCK phosphorylates cofilin in actin-meditated cytokinesis during mouse oocyte meiosis. Biol. Reprod. 90, 37.

Ferreira, C.R., Burgstaller, J.P., Perecin, F., Garcia, J.M., Chiaratti, M.R., Méo, S.C., Müller, M., Smith, L.C., Meirelles, F.V. \& Steinborn, R. (2010). Pronounced segregation of donor mitochondria introduced by bovine ooplasmic transfer to the female germ-line. Biol. Reprod. 82, $563-71$.

Förtsch, J., Hummel, E., Krist, M. \& Westermann, B. (2011). The myosin-related motor protein Myo2 is an essential mediator of bud-directed mitochondrial movement in yeast. J. Cell. Boil.194, 473-88.

Furuyama, K., Fujita, H., Nagai, T., Yomogida, K., Munakata, H., Kondo, M., Kimura, A., Kuramoto, A., Hayashi, N. \& Yamamoto, M. (1997). Pyridoxine refractory X-linked sideroblastic anemia caused by a point mutation in the erythroid 5-aminolevulinate synthase gene. Blood 90, 82230.

Hirokawa, N., Niwa, S. \& Tanaka, Y. (2010). Molecular motors in neurons: transport mechanisms and roles in brain function, development, and disease. Neuron $\mathbf{6 8 , 6 1 0 -}$ 38.

Kim, S., Kim, H.Y., Lee, S., Kim, S.W., Sohn, S., Kim, K. \& Cho, H. (2007). Hepatitis B Virus X protein induces perinuclear mitochondrial clustering in microtubule- and dynein-dependent manners. J. Virol. 81, 1714-26.

Lee, S., Kim, S., Sun, X., Lee, J.H. \& Cho, H. (2007). Cell cycle-dependent mitochondrial biogenesis and dynamics in mammalian cells. Biochem. Biophys. Res. Commun. 357, $111-7$.

Li, R. \& Albertini, D.F. (2013). The road to maturation: somatic cell interaction and self-organization of the mammalian oocyte. Nat. Rev. Mol. Cell. Biol. 14, 141-52.

Liu, S., Li, Y., Feng, H.L., Yan, J.H., Li, M., Ma, S.Y. \& Chen, Z.J. (2010). Dynamic modulation of cytoskeleton during in vitro maturation in human oocytes. Am. J. Obstet. Gynecol. 203, e1-7.

Morris, R.L. \& Hollenbeck, P.J. (1995). Axonal transport of mitochondria along microtubules and F-actin in living vertebrate neurons. J. Cell. Biol. 131, 1315-26.

Niwa, S., Tanaka, Y. \& Hirokawa, N. (2008). KIF1Bbetaand KIF1A-mediated axonal transport of presynaptic regulator Rab3 occurs in a GTP-dependent manner through DENN/MADD. Nat. Cell. Biol. 10, 126979.

Ou, X.H., Li, S., Wang, Z.B., Li, M., Quan, S., Xing, F., Guo, L., Chao, S.B., Chen, Z., Liang, X.W., Hou, Y., Schatten, H. \& Sun, Q.Y. (2012). Maternal insulin resistance causes oxidative stress and mitochondrial dysfunction in mouse oocytes. Hum. Reprod. 27. 2130-45.

Quintero, O.A., DiVito, M.M., Adikes, R.C., Kortan, M.B., Case, L.B., Lier, A.J., Panaretos, N.S., Slater, S.Q., Rengarajan, M., Feliu, M. \& Cheney, R.E. (2009). Human Myo19 is a novel myosin that associates with mitochondria. Curr. Biol. 19, 2008-13.

Ricquier, D. \& Bouillaud, F. (2000). Mitochondrial uncoupling proteins: from mitochondria to the regulation of energy balance. J. Physiol. 529, 3-10.

Schuh, M. (2011). An actin-dependent mechanism for longrange vesicle transport. Nat. Cell. Biol. 12, 1431-6.

Schuh, M. \& Ellenberg, J. (2008). A new model for asymmetric spindle positioning in mouse oocytes. Curr. Biol. 18, 1986-92.

Seabra, M.C. \& Coudrier, E. (2004). Rab GTPases and myosin motors in organelle motility. Traffic 5, 393-9.

Stojkovic, M., Machado, S.A., Stojkovic, P., Zakhartchenko, V., Hutzler, P., Goncalves, P.B. \& Wolf, E. (2001). Mitochondrial distribution and adenosine triphosphate content of bovine oocytes before and after in vitro maturation: correlation with morphological criteria and developmental capacity after in vitro fertilization and culture. Biol. Reprod. 64, 904-9.

Sun, Q.Y., Wu, G.M., Lai, L., Park, K.W., Cabot, R., Cheong, H.T., Day, B.N., Prather, R.S. \& Schatten, H. (2001). Translocation of active mitochondria during pig oocyte maturation, fertilization and early embryo development in vitro. Reproduction 122, 155-63. 
Tanaka, Y., Kanai, Y., Okada, Y., Nonaka, S., Takeda, S., Harada, A. \& Hirokawa, N. (1998). Targeted disruption of mouse conventional kinesin heavy chain, kif5B, results in abnormal perinuclear clustering of mitochondria. Cell 93, 1147-58.

Van Blerkom, J. (1991). Microtubule mediation of cytoplasmic and nuclear maturation during the early stages of resumed meiosis in cultured mouse oocytes. Proc. Natl. Acad. Sci. USA 88, 5031-5.

Viet Lin, N., Kikuchi, K., Nakai, M., Tanihara, F., Noguchi, J., Kaneko, H., Dang-Nguyen, T. Q., Thi Men, N., Van Hanh, N., Somfai, T., Nguyen, B. X., Nagai, T., and Manabe, N. (2013). Fertilization ability of porcine oocytes reconstructed from ooplasmic fragments produced and characterized after serial centrifugations. J. Reprod. Dev. 59, 549-56.

Wilding, M., Dale, B., Marino, M., di Matteo, L., Alviggi, C., Pisaturo, M. L., Lombardi, L. \& De Placido, G. (2001). Mitochondrial aggregation patterns and activity in human oocytes and preimplantation embryos. Hum. Reprod. 16, 909-17.

Yi, K., Unruh, J.R., Deng, M., Slaughter, B.D., Rubinstein, D. \& Li, R. (2011). Dynamic maintenance of asymmetric meiotic spindle position through Arp2/3-complex-driven cytoplasmic streaming in mouse oocytes. Nat. Cell. Biol. 13, 1252-8.

Yoshioka, K., Suzuki, C. \& Onishi, A. (2008). Defined system for in vitro production of porcine embryos using a single basic medium. J. Reprod. Dev. 54, 208-13.

Yu, Y., Dumollard, R., Rossbach, A., Lai, F.A. \& Swann, K. (2010). Redistribution of mitochondria leads to bursts of ATP production during spontaneous mouse oocyte maturation. J. Cell. Physiol. 224, 672-80.

Zhao, C., Takita, J., Tanaka, Y., Setou, M., Nakagawa, T., Takeda, S., Yang, H.W., Terada, S., Nakata, T., Takei, Y., Saito, M., Tsuji, S., Hayashi, Y. \& Hirokawa, N. (2001). Charcot-Marie-Tooth disease type $2 \mathrm{~A}$ caused by mutation in a microtubule motor KIF1Bbeta. Cell 105, 587-97. 